\title{
Clearing the Pediatric Cervical Spine
}

\author{
Andreas D. Weber ${ }^{1}$ Michael L. Nance ${ }^{1,2}$
}

Published online: 19 September 2016

(C) Springer International Publishing AG 2016

\begin{abstract}
Purpose of Review Management and clinical evaluation of children who present with cervical spine injuries can be challenging, particularly in the pre-verbal child and in the presence of polytrauma.

Recent Findings The successful approach to the pediatric patients' cervical spine involvement requires the following elements: an understanding of the significance of the problem, basic principles involved the management of pediatric cervical spine, and the role of radiologic studies in this patient population.

Summary This chapter highlights the common characteristics and principles behind cervical spine trauma in children, reviews the available evidence for assessment of these injuries, and focuses on a step-wise approach that can be used in the assessment and clearance of the pediatric cervical spine.
\end{abstract}

Keywords Trauma $\cdot$ Pediatric trauma $\cdot$ Cervical spine Clearance of cervical spine

\section{Introduction}

The clinical evaluation of children who present with cervical spine injuries can often be a real pain in the neck. This is especially true in those patients that lack adequate verbal

This article is part of the Topical Collection on Pediatric Trauma

Michael L. Nance

NANCE@email.chop.edu

The Children's Hospital of Philadelphia, Philadelphia, PA, USA

2 The Perelman School of Medicine at the University of Pennsylvania, Philadelphia, PA, USA skills, have an altered mental status, and/or patients that present in the setting of polytrauma. Furthermore, when injured, the maturing pediatric cervical spine may demonstrate a unique injury pattern compared with the adult cervical spine (both in terms of skeletal development and soft tissue integrity) thereby complicating the clinical picture as well as the interpretation of the radiological studies. This chapter identifies the common characteristics of pediatric cervical spine injuries as well as providing an algorithm for the step-wise approach to the assessment and clearing of the pediatric cervical spine based on the Consensus Guidelines of the Trauma Association of Canada Pediatric Committee [1••].

\section{Epidemiology}

Blunt cervical spine injury in the pediatric population is uncommon, present in only $1-2 \%$ of all pediatric trauma admissions $[2,3]$. Further, the incidence of pediatric cervical spine injuries varies by age (1.2 per 100,000/year in children less than 11 years old compared to 13.2 per $100,000 /$ year in children older than 11) [4•]. This difference is particularly important to consider during the evaluation of these patients as injury level also varies by the age of the patient [5•].

Spine injuries can be devastating to both the child and their family. Cervical spine injuries are often associated with a significant mortality (19-27\%) [6] and morbidity (e.g., up to $67 \%$ of patients with cervical spine injuries have resultant neurological deficits) [7]. Of note, upper cervical spine injuries carry a $23 \%$ mortality risk when compared to just $4 \%$ with lower cervical spine [8]. Further, life expectancy with cervical spine injuries is reduced by anywhere from 6 to 45 years depending on the level and completeness of injury [9]. Therefore, it is vitally important that the diagnosis of these 
injuries is made both quickly and with the highest accuracy to ensure the greatest possible outcome for this at-risk group.

\section{Risk Factors}

Despite ongoing vehicle design safety and child restraint systems, the most common cause of blunt cervical spine injury continues to be motor vehicle collisions. Similar to the epidemiology of cervical trauma - the risk factors associated with these injuries are correlated with age group. In addition to motor vehicle collisions, younger children are more prone to C-spine injury after falls and pedestrian accidents, whereas older children more commonly sustain cervical spine injury during contact sports and bicycling [10].

Injuries in children differ from those in the adult population. Anatomic differences that may contribute to cervical spine injuries in the pediatric patient include a higher center of gravity/fulcrum of neck motion secondary to the difference between head and body size, multiple vertebral ossification centers, and lax ligamentous structures $[5 \bullet, 8,11 \bullet \bullet$. In addition, psychosocial and developmental factors are also important to consider (e.g., young children who are beginning to walk vs. older children who engage in contact sports and other recreational activities) [12].

In addition, a number of medical conditions are also associated with cervical spine abnormalities that may predispose a patient to cervical spine injury, including Down syndrome, mucopolysaccharidosis, and achondroplasia [4•].

\section{Initial Management for Suspected Cervical Spine Injuries}

The management of patients with suspected cervical spine injuries is broken down into an acute/stabilization phase and an evaluation/clearance phase. It is important to remember that all trauma patients need spinal clearance. The possibility of a spinal cord injury should be considered in all patients that present with a traumatic mechanisms. The necessity for and extent of evaluation will vary by patient and circumstances.

Acute stabilization of these patients should include [13].

(1) Stabilization of the spine with age/size appropriate immobilization,

(2) Assessment of airway, breathing, and circulation,

(3) Ensure that pain and anxiety are managed with appropriate analgesics and sedatives [14].

Children presenting with traumatic cardiopulmonary arrest should be managed according to Advanced Trauma Life Support (ATLS) guidelines with prompt attention to gaining control of the airway while maintaining in-line manual cervical stabilization [15]. Once the airway has been secured, a rigid cervical collar should be utilized. For children who are alert and/or have more subtle signs of cervical spine injury, a rigid cervical collar alone is usually sufficient to protect against potential worsening of injury. However, this intervention is known to be associated with adverse effects including pain, pressure wounds, encumbered airway management, and respiratory compromise $[16,17]$. Therefore, the cervical spine should be cleared as soon as is clinically feasible. If clearance is delayed, a padded collar should be used.

\section{Principles of Evaluation}

Does every patient need radiographic imaging to exclude a cervical spine injury (CSI) or is it possible to clear the pediatric cervical spine clinically? Viccellio et al. evaluated the efficacy of the National Emergency X-Radiography Utilization Study (NEXUS) decision instrument for obtaining cervical spine radiography in pediatric trauma victims. The NEXUS tool relies upon the established "low-risk criteria" that were identified in this trial. If all of the criteria were negative on assessment, the NEXUS tool establishes that radiological imaging is very unlikely to be necessary.

Low-risk criteria include ABSENCE of:

- Midline cervical tenderness,

- Intoxication,

- Altered level of consciousness,

- Focal neurological deficit,

- Distracting injury.

Viccellio et al. stated that the NEXUS decision instrument performed well (overall) in children but advised caution in its use for the infant and toddler age group because of the relatively small number of children enrolled during the study of that age group $[11 \bullet \bullet]$.

Overall, the NEXUS trial $(N=34,069)$ included a diverse sample, including 3065 children who were younger than 18 years, of whom $30(0.98 \%)$ sustained a cervical spinal injury. Patients that were considered "low-risk" (19.7\%) had no cervical spine injuries identified. Applying the NEXUS criteria to the pediatric population, the sensitivity and negative predictive value for this algorithm for CSI was $100 \%$, the specificity was $19.9 \%$, and the positive predictive value was $1.2 \%$. As previously mentioned, due to the small number of infants and toddlers in this study (6\%), use caution applying NEXUS rules to that group. It should be noted that meeting low-risk criteria was more likely in patients between birth and 8 years old $(26.5 \%)$ when compared to the older pediatric population (9-18 years old, $18.5 \%$ ). To date, follow up studies have failed to replicate the sensitivity that was obtained in the NEXUS trial. 
To evaluate whether simple a set of clinical criteria could be safely utilized to rule out CSI in patients younger than 3 years, Pieretti-Vanmarcke et al. [18] collected data from 22 pediatric trauma centers $(N=12,537$ screened, $N=83(0.66 \%)$ suffered cervical spine injuries).

In this population, four independent predictors of risk for CSI were identified:

- Glasgow Coma (total) Score $<14$,

- Glasgow Coma Score of the eye (specifically) $=1$,

- Motor vehicle crash, and

- Age 2 years or older.

The authors formulated a weighted score metric by assigning a value to each independent predictor based to its magnitude of effect (either 1, 2, or 3 points). As such, a Glasgow Coma Scale (GCS) score of less than 14 (3 points), GCS eye score of 1 (2 points), MVC mechanism (2 points), and age 2 years or older (25-36 months; 1 point). In their analysis, patients with a documented score of $<2(N=8707$ patients, $69.5 \%$ ) were eligible for cervical spine clearance without imaging. In addition, a net score of 0 or 1 had a negative predictive value of $99.93 \%$ successfully excluding the presence of CSI. Their analysis concluded a sensitivity of almost $93 \%$ (sensitivity $=69.9 \%$ ) indicating that their model was an excellent fit for predicting CSI. A score of 2 or 3 did not mandate special imaging, but rather could not exclude the presence of a CSI.

In a separate study, Leonard et al. conducted a case-control study $(N=540)$ that identified an 8 -variable model for determining cervical spine injury in children after blunt trauma:

- Altered mental status

- Focal neurologic deficit

- Complaint of neck pain

- Torticollis

- Significant torso injury

- Predisposing conditions (e.g., Down syndrome)

- High risk mechanism: diving

- High risk mechanism: MVC

Having one or more of the aforementioned factors was found to be $98 \%$ sensitive (and $26 \%$ specific) for diagnosing cervical spine injury [16].

In pediatric patients whose cervical spine cannot be cleared based on clinical examination, management of these patients should be undertaken with a goal of minimizing unnecessary radiation exposure. This is primarily due to the increased risk of developing radiation-induced malignancy later in life.

Risk estimates for radiation-induced malignancy are primarily derived from the mortality data that was obtained from Japanese WW-II survivors who were exposed in Hiroshima and Nagasaki [19]. Studies have shown strong evidence that cancer mortality risk increases with increasing radiation doses. In addition, due rapid turnover of the growing cells in the child, younger patients are at greater risk of radiationinduced malignancies [19, 20]. There is good evidence of increased risk for cancer mortality risk at doses between 50 and $100 \mathrm{mSv}$ (with additional evidence suggesting that cancer mortality risk is increased in doses as low as $10-50 \mathrm{mSv}$ ) $[1 \bullet \bullet$. In the typical pediatric trauma patient, the mean radiation exposure was $12-14 \mathrm{mSv}$, with the vast majority a result of CT scanning [21•]. As such, efforts to minimize radiation, and in particular CT scanning, is beneficial.

While cervical spine clearance is necessary in all trauma patients, in most a reliable clinical examination will suffice. In those in whom a reliable exam is not possible but the suspicion is low, one can consider re-examination at a later time (e.g., the next morning for an admitted patient) to see if a reliable examination can be obtained. The routine use of CT scanning should be discouraged in the pediatric population in favor of exam directed imaging. In an attempt to consolidate the available risk factor data and formulate a methodology for assessing CSI that could be easily employed by trauma physicians - the Trauma Association of Canada Pediatric Committee formulated the C-Spine Evaluation Pathway that was published by Chung et al. [1••].

\section{Pediatric Cervical Spine Clearance Algorithm}

Based on the patients GCS, the pathway can be divided either by reliable clinical exam or unreliable clinical exam [1••].

\section{"Reliable Clinical Examination" Pathway}

For patients awake and alert with a GCS equal 15, the "Reliable Clinical Examination" pathway (Fig. 1) guides the decision making process: The cervical spine can be clinically cleared and the collar discontinued if the patient meets the NEXUS criteria and is able to perform flexion/extension the neck and rotate the head $45^{\circ}$ to both sides without pain.

If this is not the case, conventional radiological (AP/lateral/ odontoid x-rays for cooperative and only AP/lateral x-rays for uncooperative patients) and neurological examination will guide further decisions. If the neurological exam is abnormal, the patient must remain in collar; however, it should be changed to a padded cervical spine collar as soon as is clinically appropriate. Radiological imaging utilizing MRI (and possibly CT of the C-spine) is needed and the spine service should be consulted. If neurological and conventional radiological exam are normal (as well as adequate XR films obtained), reexamination is recommended, particularly in children older than 8 years.

However, if the child is 8 years old or younger and a head CT is planned, it is recommended to include a CT scan of the 
Fig. 1 Reliable clinical exam pathway derived from Trauma Association of Canada consensus guidelines $[1 \bullet \bullet]$ (used with permission)

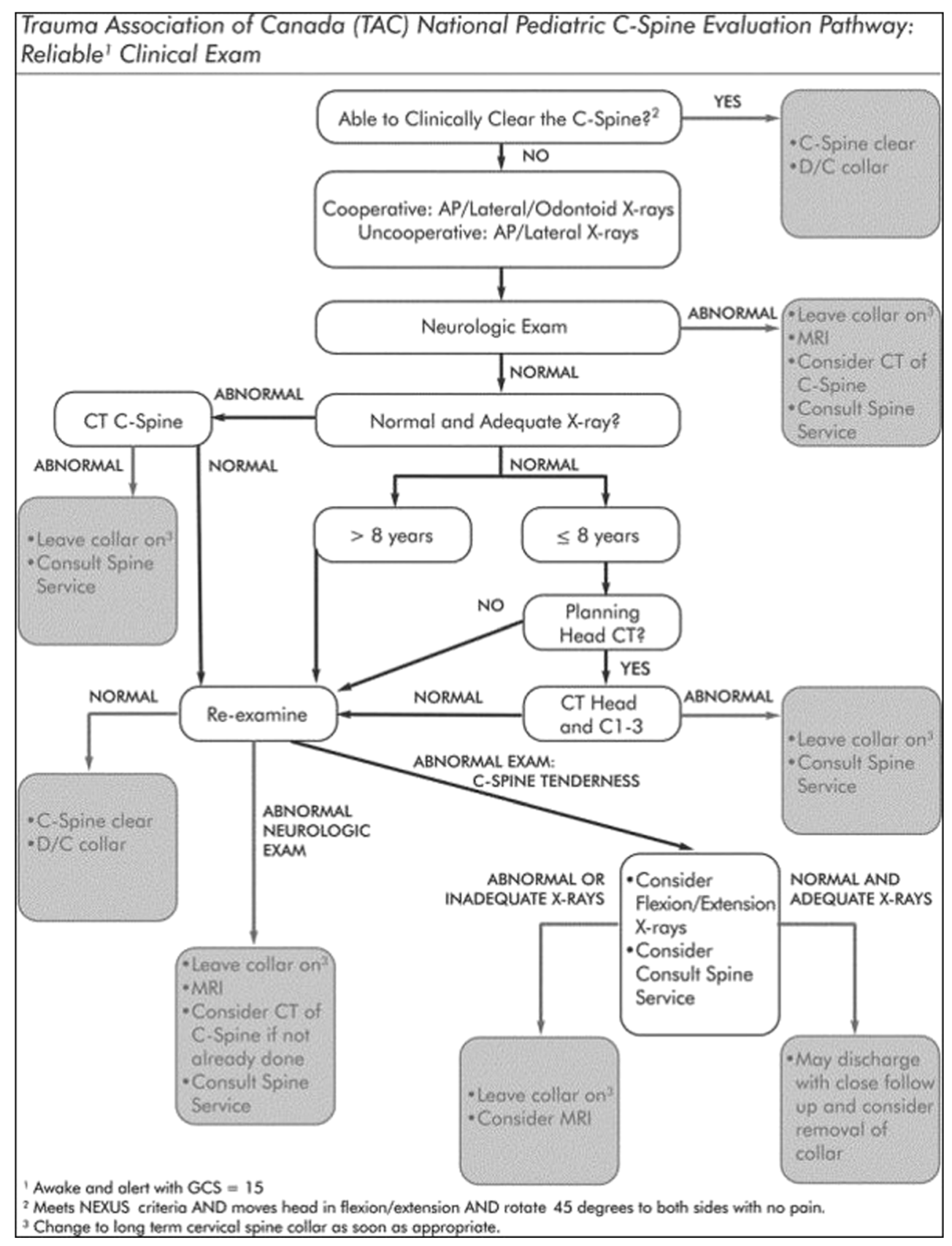

upper c-spine (C1-3), to prevent the entire c-spine from being irradiated. This differentiation based on age has the goal of not missing an injury as this particular age group is at highest risk for upper c-spine injury. That said, those patients with abnormal CT findings need to remain with the collar on, as well as consultation with the spine service is recommended.

Normal findings on the other hand need physical re-examination. If the examination shows any cervical spine tenderness, consideration should be given for Flexion/Extension $\mathrm{x}$ rays and/or spine service consultation. In case of normal and adequate $\mathrm{x}$-rays, it may be appropriate to discharge the patient as long as close follow-up can be organized.
Patients with abnormal or inadequate $\mathrm{x}$-rays need to remain in the collar and an MRI should be considered. Patients presenting with any abnormality on neurological re-examination require further evaluation with $\mathrm{MRI}$ as well as consideration of CT of the cervical spine (if not already performed) in cervical spine protection with collar and consultation with the spine service.

If the re-examination is normal, the cervical spine can be cleared and the collar removed.

Most importantly, patients with abnormal findings on the $\mathrm{CT}$ at any point in the algorithm need further assessment by the spine service. 


\section{“Unreliable Clinical Examination” Pathway}

The "Unreliable Clinical Examination" pathway (Fig. 2) was specifically formulated for patients who present in either an unconscious state or demonstrate a decreased level of consciousness (with a corresponding GCS of less than 15).

Any abnormality on the neurological examination requires the same steps as the reliable patients with abnormal neurological exam: The patient has to remain in the collar, a MRI must be performed, and the spine service needs to be consulted. A CT of the C-spine can be considered; however, it is important to remember that, a CT is not definitive enough to remove the cervical collar - even if the results were normal.

That said, even if the neurological examination is normal, the collar has to be left on and the patient requires additional radiological imaging such as AP and lateral X-rays. At this juncture, the cervical spine service has to be involved for any abnormal findings. It is important to consider thoraco-lumbar spine co-injuries if the patients shows cervical spine injuries.

If neurological and radiological evaluations are normal, frequent reassessment of level of consciousness is recommended during the first 24-72 h. If the level of consciousness does not improve, the spine service should be involved and a MRI of the cervical spine is recommended. However, if the patient is alert and cooperative, the "Reliable Clinical Examination" can be followed.

\section{Conclusions}

It is important to remember that all trauma patients require spinal clearance. The process of clearance, especially in the pediatric patient, can be complex and demanding. However, in this chapter, we have attempted to parse the most relevant information in a clear and concise manner towards balancing
Fig. 2 Unreliable clinical exam pathway derived from Trauma Association of Canada consensus guidelines $[1 \bullet \bullet]$ (used with permission)

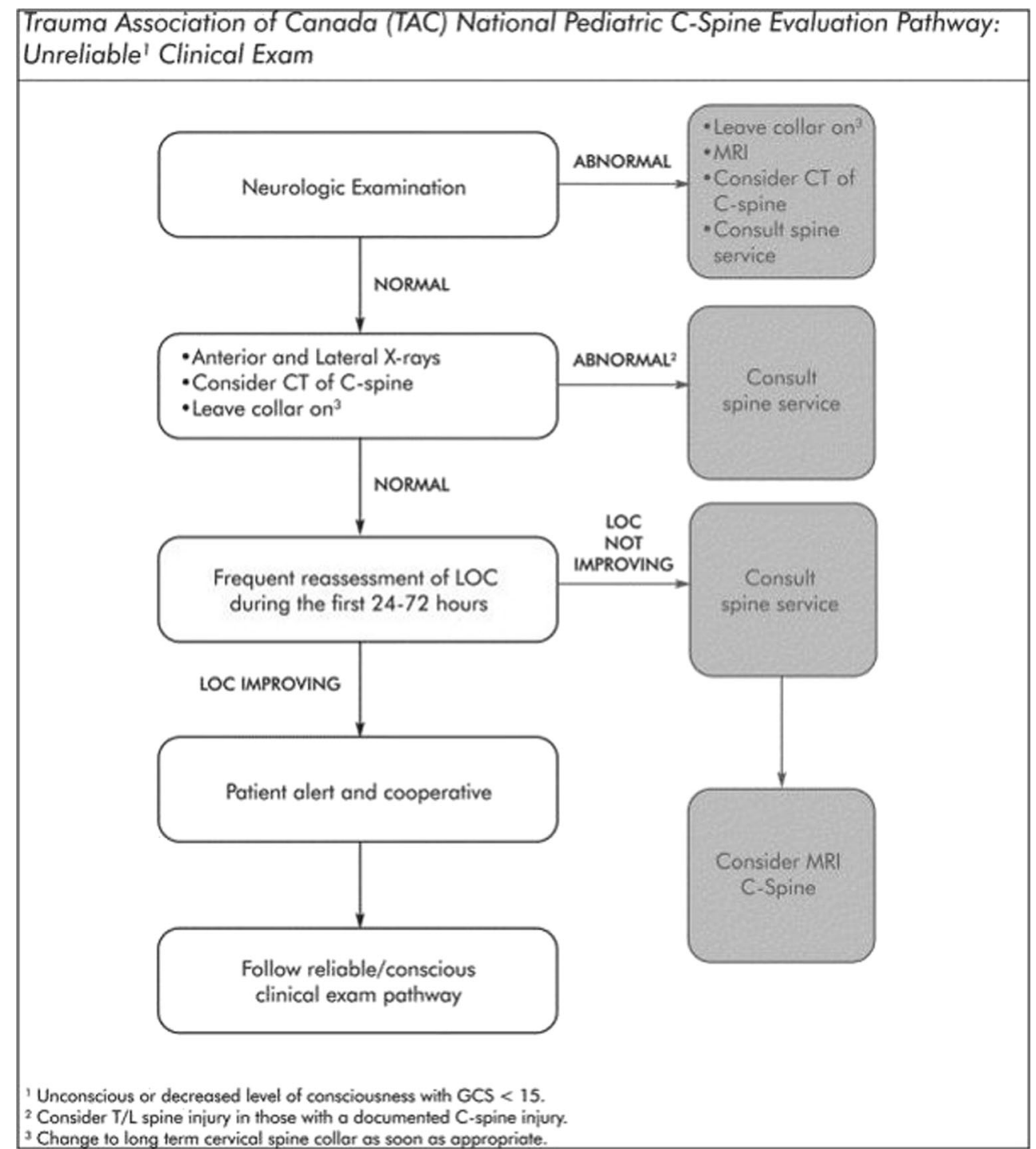


the needs of increased accuracy in timely diagnosis of C-Spine injuries with a corresponding decrease in radiological imaging. The guidelines discussed herein serve the additional purpose of decreasing both healthcare costs and patient-related radiological exposure while maintaining the highest levels of care.

Therefore, it is our belief that the pathways formulated by the Trauma Association of Canada Pediatric Committee accomplish these (aforementioned) goals and are the principle reasons why institutions throughout Canada and certain trauma centers in the USA employ them.

\section{Compliance with Ethical Standards}

Conflict of Interest Andreas D. Weber and Michael L. Nance declare that they have no conflicts of interest.

Human and Animal Rights and Informed Consent This article does not contain any studies with human or animal subjects performed by any of the authors.

\section{References}

Papers of particular interest, published recently, have been highlighted as:

- Of importance

•- Of major importance

1.• Chung S, Mikrogianakis A, Wales PW, et al. Trauma association of Canada Pediatric Subcommittee National Pediatric Cervical Spine Evaluation Pathway: consensus guidelines. J Trauma. 2011;70: 873-84. Summary of the working group of the Trauma Association of Canada that highlights the available literature and provides an algorithm for clearance of the pediatric cervical spine.

2. Platzer P, Jaindl M, Thalhammer G, et al. Cervical spine injuries in pediatric patients. J Trauma. 2007;62:389-96.

3. Brown RL, Brunn MA, Garcia VF. Cervical spine injuries in children: a review of 103 patients treated consecutively at a level I pediatric trauma center. J Pediatr Surg. 2001;36:1107-14.

4. Leonard JR, Jaffe DM, Kuppermann N, et al. Cervical spine injury patterns in children. Pediatrics. 2014;133(5):e1179-88. Large, multicenter review on the anatomic pattern of injury and injury mechanisms for pediatric cervical spine injuries.

5. Leonard JC, Jaffe DM, Olsen CS, Kuppermann N. Age-related differences in factors associated with cervical spine injuries in children. Acad Emerg Med. 2015;22:441-6. Age-based review of factors that impact the distribution of cervical spine injuries in children.

6. Polk-Williams A, Carr BG, Blinman TA, Masiakos PT, Wiebe DJ, Nance ML. Cervical spine injury in young children: a National Trauma Data Bank review. J Pediatr Surg. 2008;43:1718-21.

7. Kanwar R, Delasobera BE, Hudson K, Frohna W. Emergency department evaluation and treatment of cervical spine injuries. Emerg Med Clin North Am. 2015;33(2):241-82.

8. Patel JC, Tepas III JJ, Mollitt DL, Pieper P. Pediatric cervical spine injuries: defining the disease. J Pediatr Surg. 2001;36:373-6.

9. National Spinal Cord Injury Statistical Center. 2011 NSCISC annual statistical report-complete public version. Birmingham: University of Alabama; 2011.

10. Kokoska ER, Keller MS, Rallo MC, Weber TR. Characteristics of pediatric cervical spine injuries. J Pediatr Surg. 2001;36:100-5.

11.• Viccellio P, Simon H, Pressman BD, et al. A prospective multicenter study of cervical spine injury in children. Pediatrics. 2001;108:e20 5. The pediatric component of the large NEXUS cervical spine trial.

12. Parent S, Mac-Thiong JM, Roy-Beaudry M, Sosa JF, Labelle H. Spinal cord injury in the pediatric population: a systematic review of the literature. J Neurotrauma. 2011;28:1515-24.

13. Walters BC, Hadley MN, Hurlbert RJ, et al. Guidelines for the management of acute cervical spine and spinal cord injuries: 2013 update. Neurosurgery. 2013;60 Suppl 1:82-91.

14. Hurlbert RJ, Walters BC, Hadley MN, et al. Pharmacological therapy for acute spinal cord injury. Neurosurgery. 2015;76 Suppl 1: S71-83.

15. Committee on Trauma, American College of Surgeons. ATLS: advanced trauma life support program for doctors. 9th ed. Chicago: American College of Surgeons; 2013.

16. Leonard JC, Kuppermann N, Olsen C, et al. Factors associated with cervical spine injury in children after blunt trauma. Ann Emerg Med. 2011;58:145-55.

17. Linares H, Mawson A, Suarez E, et al. Association between pressure sores and immobilization in the immediate post-injury period. Orthopedics. 1987;10:571-3.

18. Pieretti-Vanmarke R, Velmahos GC, Nance ML, et al. Clinical clearance of the cervical spine in blunt trauma patients younger than 3 years: a multi-center study of the American Association for the Surgery of Trauma. J Trauma. 2009;67:543-50.

19. Brenner D, Elliston C, Hall E, Berdon W. Estimated risks of radiation-induced fatal cancer from pediatric CT. AJR Am J Roentgenol. 2001;176(2):289-96.

20. Pearce MS, Salotti JA, Little MP, McHugh K, Lee C, Kim KP, et al. Radiation exposure from CT scans in childhood and subsequent risk of leukaemia and brain tumours: a retrospective cohort study. Lancet. 2012;380(9840):499-505.

21. Kim PK, Zhu X, Houseknecht E, Nickolaus D, Mahboubi S, Nance ML. Effective radiation dose from radiologic studies in pediatric trauma patients. World J Surg. 2005;29(12):1557-62. Single-institutional review of imaging practices for pediatric trauma patients highlighting the predominance of $\mathrm{CT}$ scanning and radiation exposure in evaluation of this population. 